ORIGINAL ARTICLE

\title{
Determinação de resíduo de bifentrina em banana após a colheita e na água de lavagem
}

\section{Determination of bifenthrin residue in bananas after harvest and in washing water}

\section{Ariane Castricini ${ }^{1 *}$ (D), Ana Luiza Santos de Oliveira², Ane Patrícia Cacique ${ }^{2}$, Maria Geralda Vilela Rodrigues ${ }^{1}$, Flaviano Oliveira Silvério ${ }^{2}$}

\author{
${ }^{1}$ Empresa de Pesquisa Agropecuária de Minas Gerais (EPAMIG), Campo Experimental do Gorutuba, Nova \\ Porteirinha/MG - Brasil \\ ${ }^{2}$ Universidade Federal de Minas Gerais (UFMG), Instituto de Ciências Agrárias, Montes Claros/MG - Brasil \\ *Corresponding Author: Ariane Castricini, Empresa de Pesquisa Agropecuária de Minas Gerais (EPAMIG), \\ Campo Experimental do Gorutuba, Rodovia MGT 122, km 155, CEP: 39525-000, Nova Porteirinha/MG - Brasil, \\ e- mail: ariane@epamig.br
}

Cite as: Castricini, A., Oliveira, A. L. S., Cacique, A. P., Rodrigues, M. G. V., \& Silvério, F. O. (2021). Determination of bifenthrin residue in bananas after harvest and in washing water. Brazilian Journal of Food Technology, 24, e2020118. https://doi.org/10.1590/1981-6723.11820

\begin{abstract}
Resumo
Na bananicultura, o uso de sacos impregnados por inseticida e a lavagem das pencas são técnicas que visam à qualidade dos frutos. Objetivou-se monitorar, por cromatografia em fase gasosa acoplada à espectrometria de massas, resíduo de bifentrina em bananas lavadas e não lavadas em tanques, assim como na água de lavagem dos frutos. Desde a emissão até a colheita, os cachos foram protegidos com sacos impregnados por bifentrina a $1,0 \mathrm{~g} \mathrm{~kg}^{-1}$, os quais foram retirados após a colheita, para despencamento e lavagem ou não das pencas em tanques com água, detergente e sulfato de alumínio. Os métodos Quick, Easy, Cheap, Effective, Rugged, Safe Method - QuEChERS e Extração Líquido - Líquido com Partição a Baixa Temperatura - ELL - PBT foram utilizados para extrair resíduo de bifentrina dos frutos no ponto de colheita e maduros, e da água, respectivamente. As porcentagens de recuperação de bifentrina foram $88,16 \%, 103,4 \%$ e $89,32 \%$, na casca, na polpa e em casca + polpa, respectivamente, atendendo à legislação brasileira. No ponto de colheita, na casca e na casca + polpa dos frutos lavados, detectou-se $0,060 \mathrm{mg} \mathrm{kg}^{-1} \mathrm{e}$ $0,010 \mathrm{mg} \mathrm{kg}^{-1}$, respectivamente. Quando não lavados, a concentração de bifentrina, na casca, foi de $0,31 \mathrm{mg} \mathrm{kg}^{-1} \mathrm{e}$ de $0,16 \mathrm{mg} \mathrm{kg}^{-1}$, na casca + polpa. Não foi detectado resíduo de bifentrina na polpa, independente do estádio de maturação e lavagem, tampouco na água de lavagem. Em frutos maduros não lavados, foram quantificado $0,10 \mathrm{mg} \mathrm{kg}^{-1}$ e $0,040 \mathrm{mg} \mathrm{kg}^{1}$ de bifentrina na casca e em casca + polpa, respectivamente. A lavagem não elimina, mas reduz o resíduo de bifentrina na casca dos frutos no ponto de colheita e, quando maduros, somente os não lavados apresentam resíduo.
\end{abstract}

Palavras-chave: Musa sp.; Piretroide; Extração; Cromatografia gasosa; Casca; Polpa.

\section{Abstract}

In banana farming, the use of bags impregnated by insecticides and the washing of bunches are techniques that aim at maintaining the quality of fruits. This study aimed to monitor, by Gas Chromatography (GC) coupled with Mass Spectrometry (MS), bifenthrin residue in washed and unwashed bananas, as well as in the fruit washing water. 
From the emission until the harvest, the bunches were protected with bags impregnated with bifenthrin at $1.0 \mathrm{~g} \mathrm{~kg}^{-1}$, which were removed after harvest for fruit dropping and washing or not bunches in tanks with water, detergent and aluminum sulfate. The Quick, Easy, Cheap, Effective, Rugged, Safe Method - QuEChERS and liquid-liquid extraction with the low temperature partition method were used to extract bifenthrin residue from fruits at harvest point and ripe, and from water, respectively. Bifenthrin recovery percentages were $88.16 \%, 103.4 \%$ and $89.32 \%$ in peel, pulp and in peel + pulp, respectively, in compliance with Brazilian legislation. At the harvest point, $0.060 \mathrm{mg} \mathrm{kg}^{-1}$ and $0.010 \mathrm{mg} \mathrm{kg}^{-1}$ were detected in peel and in peel + pulp of washed fruits, respectively. When unwashed, bifenthrin concentration in peel was $0.31 \mathrm{mg} \mathrm{kg}^{-1}$ and $0.16 \mathrm{mg} \mathrm{kg}^{-1}$ in peel + pulp. No bifenthrin residue was detected in pulp, regardless of maturation stage and washing, as well as in the washing water. In mature unwashed fruits, $0.10 \mathrm{mg}$ $\mathrm{kg}^{-1}$ and $0.040 \mathrm{mg} \mathrm{kg}^{-1}$ of bifenthrin were detected in peel and in peel + pulp, respectively. Washing does not eliminate the residues, but reduces bifenthrin residue in fruit peel at the harvest point; when ripe, only unwashed fruits showed presence of bifenthrin residue.

Keywords: Musa sp.; Pyrethroid; Extraction; Gas chromatography (GS); Peel; Pulp.

\section{Introdução}

No cenário da fruticultura brasileira, a banana (Musa spp.) foi a segunda fruta mais produzida em 2018, com 6.752,171 ton (Instituto Brasileiro de Geografia e Estatística, 2018). A bananicultura nacional está presente em todas as regiões, mas destaca-se nas regiões Sudeste e Nordeste, com 2.313,788 ton e 2.259,288 ton, respectivamente. Nessas regiões, as áreas mais representativas têm sido o litoral sul paulista, o norte de Minas Gerais e o sul da Bahia (Instituto Brasileiro de Geografia e Estatística, 2018).

Durante a produção, colheita e pós-colheita da banana, algumas técnicas têm sido empregadas com o objetivo de minimizar ou impedir a ocorrência de danos nos frutos. Estes são frágeis e qualquer avaria sofrida antes ou após a colheita, enquanto verdes, torna-se visível durante o amadurecimento, o que reduz o valor comercial e interfere negativamente na qualidade do fruto.

Os insetos C. bicinctus, C. orchidii e T. lineatus, conhecidos como tripes-da-ferrugem-das-frutas ou ferrugem-dos-frutos, são os responsáveis pelos sintomas conhecidos como ferrugem-dos-frutos em bananais. Nessa condição, ocorrem danos consideráveis à aparência externa dos frutos, sem prejudicar a polpa, mas depreciando-os para a comercialização, especialmente para a exportação (Brasil, 2019). Entre as medidas de controle, está a proteção dos cachos no campo, ainda durante o desenvolvimento dos frutos, com sacos impregnados internamente com inseticida, como a bifentrina, que é um piretroide sintético, pouco solúvel em água $\left(0,1 \mathrm{mg} \mathrm{L}^{-1}\right)$, com elevada estabilidade à luz natural e em meio aquoso (Freitas, 2013).

Após a colheita dos cachos de banana, os sacos utilizados no ensacamento são retirados e se procede ao despencamento e à imersão das pencas em tanques de lavagem. A lavagem é feita com a finalidade de coagular o látex (nódoa ou cica), que exsuda da região do corte no momento do despencamento, e para retirada de sujeiras aderidas nos frutos. O látex não removido pode causar queimaduras na casca do fruto, que se manifestam durante o amadurecimento, em forma de manchas escuras, depreciando-o. Sulfato de alumínio e detergente neutro são adicionados à água nos tanques de lavagem, para cicatrização e limpeza dos frutos, respectivamente.

Embora a lavagem das pencas seja uma atividade de rotina, indispensável para manutenção da qualidade pós-colheita da banana, ainda há uma lacuna de informação sobre sua eficácia na remoção de resíduo de agrotóxicos, como a bifentrina. Entretanto, em frutos produzidos sob ensacamento e que não passaram pela etapa de lavagem após a colheita, Castricini et al. (2019) verificaram que as concentrações médias de bifentrina na casca variaram entre $0,34 \mathrm{mg} \mathrm{e} 0,10 \mathrm{mg} \mathrm{kg}^{-1}$, a depender do estádio de maturação, ou seja, do tempo percorrido entre a colheita e o consumo. Segundo os autores, não foi detectado resíduo na polpa dos frutos em nenhum estádio de maturação. 
A determinação da concentração do resíduo de bifentrina em bananas produzidas em cachos ensacados, tanto no ponto de colheita quanto maduras, e na água de lavagem, torna-se relevante sob o ponto de vista de saúde do consumidor e ambiental. Segundo Miyamoto et al. (1995), os inseticidas piretroides, quando ingeridos, podem ser absorvidos pelo trato gastrointestinal e metabolizados no fígado, por meio de reações de oxidações e hidrólise de ésteres. No sistema nervoso, impedem a transmissão do impulso nervoso, levando à indução de atividades repetitivas e duradouras, como tremores, salivação excessiva, hipersensibilidade, convulsões, entre outras.

A presença de resíduos de agrotóxicos acima dos limites permitidos ou em culturas nas quais sua utilização não é autorizada pode originar não somente problemas de saúde pública e de ordem ecológica como também barreira comercial, tanto no mercado interno como no externo (Oviedo et al., 2003). Além, da elevada exigência dos consumidores, que ocasiona o aumento na demanda por produtos isentos de resíduos de agrotóxicos (Tiwari et al., 2010; Wakil et al., 2013).

Neste sentido, objetivou-se monitorar, por cromatografia em fase gasosa acoplada à espectrometria de massas, resíduo de bifentrina em bananas lavadas e não lavadas em tanques, assim como na água de lavagem dos frutos.

\section{Material e métodos}

\subsection{Preparo da solução padrão e fortificação das amostras}

As soluções padrão estoque de bifentrina $(92,2 \% \mathrm{~m} / \mathrm{m})$ - FMC do Brasil, foram preparadas em acetonitrila na concentração de $500 \mathrm{mg} \mathrm{L}^{-1}$ e armazenadas em temperatura de $4{ }^{\circ} \mathrm{C}$. A partir das soluções estoque, foi preparada a solução de trabalho contendo o piretroide na concentração de $50 \mathrm{mg} \mathrm{L}^{-1}$.

Acetonitrila grau HPLC, acetato de sódio e sulfato de magnésio com graus de pureza superiores a 99\% $(\mathrm{m} / \mathrm{m})$ foram adquiridos da Vetec (Rio de Janeiro, Brasil). Para o preparo de amostras, utilizou-se vórtex da Phoenix (São Paulo, Brasil); centrífuga da Kindly (São Paulo, Brasil); estufa da Ethik Technology (São Paulo, Brasil), e balança analítica da Shimadzu (São Paulo, Brasil). A solução padrão é utilizada para a obtenção do cromatograma relativo ao analito estudado e das porcentagens de recuperação do analito, nas condições cromatográficas estipuladas.

A etapa de fortificação é a adição de uma concentração conhecida do analito (bifentrina) à amostra, para posterior recuperação do mesmo. Trata-se de um ensaio normalmente utilizado para validação do método analítico a fim de estimarem-se a exatidão e a precisão dos dados. Para a fortificação, foram utilizadas bananas provenientes de cultivo sem agrotóxico e sem ensacamento dos cachos. As amostras divididas em casca, polpa e casca + polpa foram fortificadas com bifentrina na concentração de $5 \mathrm{mg} \mathrm{L}^{-1}$ e deixadas em repouso por $3 \mathrm{~h}$, para permitir a interação da bifentrina com a matriz e a evaporação do solvente.

\subsection{Análises cromatográficas}

As análises foram realizadas em cromatógrafo a gás da Agilent Technologies (GC 7890A) acoplado a um detector de massas (MS 5975C), com coluna capilar DB-5 MS (Agilent Technologies) com fase estacionária $5 \%$ difenil e 95\% dimetilpolisiloxano (30 m comprimento $\times 0,25 \mathrm{~mm}$ diâmetro interno $\times 0,25 \mu \mathrm{m}$ espessura do filme interno). A programação de temperatura do forno iniciou em $150{ }^{\circ} \mathrm{C}(1 \mathrm{~min})$ com taxa de aquecimento de $15^{\circ} \mathrm{C} \mathrm{min}^{-1}$ até $220^{\circ} \mathrm{C}$, permanecendo nesta temperatura por $1 \mathrm{~min}$. Em seguida, uma taxa de aquecimento de $30{ }^{\circ} \mathrm{C} \mathrm{min}^{-1}$ até $290{ }^{\circ} \mathrm{C}$ por $4 \mathrm{~min}$. A análise apresentou tempo total de $14 \mathrm{~min}$. $\mathrm{O}$ hélio $\left(99,9999 \%\right.$ de pureza) foi utilizado como gás de arraste a uma taxa de $1 \mathrm{~mL} \mathrm{~min}{ }^{-1}$. A temperatura do injetor split/splitless foi mantida a $280^{\circ} \mathrm{C}$. O volume da amostra injetado foi de $1 \mu \mathrm{L}$ no modo de injeção sem divisão de fluxo (splitless), utilizando autoinjetor Combi PAL. O espectrômetro de massas foi operado no modo de ionização por impacto de elétrons a $70 \mathrm{eV}$. A interface foi mantida a $300^{\circ} \mathrm{C}$ e a fonte de íons, a $230{ }^{\circ} \mathrm{C}$. O 
controle do instrumento e a aquisição de dados foram feitos com o software ChemStation (E.02.02.1431 copyright (C) 1989-2011) da Agilent Technology. As análises foram realizadas no modo monitoramento de íons seletivo (MIS). Os íons selecionados para análise foram 303,9; 305,9 e 307,9 m/z.

Para todas as amostras avaliadas, foram utilizadas as mesmas condições cromatográficas, conforme descrito no item acima.

\subsection{Determinação de resíduo de bifentrina em banana e na água de lavagem dos frutos}

Para este estudo, foram utilizadas bananas 'Grande Naine' (AAA) pertencentes ao grupo Cavendish, produzidas em área experimental da Epamig Norte, no Campo Experimental do Gorutuba (CEGR), Nova Porteirinha-MG, com as seguintes coordenadas geográficas: latitude de $15^{\circ} 46^{\prime} 38,29^{\prime \prime} \mathrm{S}$, longitude de $43^{\circ} 17^{\prime} 22,9^{\prime \prime} \mathrm{W}$, com altitude média de $500 \mathrm{~m}$ e precipitação pluvial média anual de $800 \mathrm{~mm}$, sendo o clima classificado com Aw (tropical de savana), segundo Köppen.

Após a emissão do cacho até o momento da colheita, foram utilizados sacos de proteção, internamente impregnados com bifentrina a $1,0 \mathrm{~g} \mathrm{~kg}^{-1}$, com a finalidade de controlar pragas e proteger os frutos contra queimaduras de sol e outros danos mecânicos.

Ao atingirem o ponto de colheita, dois cachos foram colhidos aleatoriamente na área de produção e, após a colheita, procedeu-se à retirada dos sacos impregnados e ao despencamento. As pencas 1,2 e 3 do primeiro cacho passaram pela etapa de lavagem de rotina em tanques contendo água, sulfato de alumínio e detergente neutro, e as pencas 1, 2 e 3 do segundo cacho não foram lavadas.

As pencas foram encaminhadas para o Laboratório de Pesquisa em Agroquímica (LPA) do Instituto de Ciências Agrárias da Universidade Federal de Minas Gerais, em Montes Claros-MG, para as etapas de análise de resíduo de bifentrina, em dois estádios de maturação da fruta proveniente das pencas lavadas e não lavadas. Os estádios de maturação foram 1 e 7, de acordo com a escala de Loesecke, utilizada pelas Normas de Classificação da Ceagesp (Companhia de Entrepostos e Armazéns Gerais de São Paulo, 2006). O estádio 1 corresponde aos frutos no ponto de colheita, ou seja, no ponto de maturidade comercial, com máximo crescimento, ainda ligeiramente quinados, e com casca totalmente verde. $\mathrm{O}$ estádio 7 corresponde a frutos maduros e aptos para o consumo. O intervalo entre os dois estádios foi de 14 dias e, nesse período, as bananas permaneceram em bandejas, sobre bancada, em temperatura ambiente.

Em ambos os estádios de maturação, três frutos foram retirados aleatoriamente de cada uma das pencas, lavadas ou não, compondo assim três repetições de três frutos por parcela experimental. Os frutos foram descascados e as amostras das matrizes casca, polpa e casca + polpa foram separadas para posterior extração e detecção do resíduo de bifentrina. As amostras foram homogeneizadas, sendo a casca picada e a polpa macerada em gral e pistilo.

A extração de bifentrina das amostras de casca, polpa e de casca + polpa de banana foi realizada pelo método QuEChERS (Quick, Easy, Cheap, Effective, Rugged, Safe Method) modificado (Carneiro et al., 2013). Em um tubo de polipropileno $(50 \mathrm{~mL})$, foram adicionados $10 \mathrm{~g}$ da amostras e $10 \mathrm{~mL}$ de acetonitrila (ACN) acidificada com ácido acético $1 \%(\mathrm{v} / \mathrm{v})$ com agitação em vórtex por $1 \mathrm{~min}$. Em seguida, foram adicionados 4,0 $\mathrm{g}$ de sulfato de magnésio anidro e 1,0 $\mathrm{g}$ de acetato de sódio, com homogeneização em vórtex por $1 \mathrm{~min}$. O sistema foi centrifugado a $4.000 \mathrm{rpm}$ por 9 min e $2 \mathrm{~mL}$ do sobrenadante foram transferidos para tubo cônico de $15 \mathrm{~mL}$, contendo 1,5 g de sulfato de magnésio anidro. Essa mistura foi novamente agitada em vórtex por $1 \mathrm{~min}$, com posterior centrifugação (4.000 rpm) por $9 \mathrm{~min}$. O volume de $1 \mathrm{~mL}$ do sobrenadante foi transferido para vial de injeção e analisado em cromatógrafo a gás acoplado ao espectrômetro de massas (CG-EM).

Os tratamentos deste estudo foram compostos pela combinação em fatorial $2 \times 3$, em cada estádio de maturação. A lavagem das pencas constituiu o primeiro fator (lavadas ou não lavadas) e a matriz avaliada (casca, polpa, casca + polpa), o segundo fator. Utilizou-se o delineamento inteiramente casualizado, com três 
repetições de três frutos para cada matriz avaliada, em cada condição de lavagem, nos distintos estádios de maturação. A ANOVA e o teste Tukey foram realizados no software SAEG 9.1 (Universidade Federal de Viçosa, 2007).

Em geral, no procedimento de lavagem, as pencas de banana passam por dois tanques, em sequência. A presença de resíduo nessa água é importante, uma vez que será descartada ou reutilizada, podendo configurar agente poluente na propriedade. Três amostras de $500 \mathrm{~mL}$ da água utilizada na lavagem das pencas foram coletadas no primeiro tanque de lavagem e, em seguida, encaminhadas para análise de resíduo de bifentrina no LPA.

A extração da bifentrina a partir da amostra de água oriunda do tanque de lavagem das bananas foi realizada utilizando-se o método de Extração Líquido - Líquido com Partição a Baixa Temperatura (ELL - PBT) (Silvério et al., 2012). Em vial de $22 \mathrm{~mL}$, foram adicionados $4 \mathrm{~mL}$ de água do tanque e $8 \mathrm{~mL}$ de ACN. Procedeu-se à agitação em vórtex por $1 \mathrm{~min}$. Em seguida, o sistema foi deixado em repouso a -18 ${ }^{\circ} \mathrm{C}$ por $1 \mathrm{~h}$, para o congelamento da fase aquosa. A fase orgânica composta pela bifentrina e acetonitrila foi filtrada em funil analítico contendo sulfato de sódio anidro. O filtrado foi recolhido em balão volumétrico de $10 \mathrm{~mL}$ e o menisco foi aferido com ACN. A amostra foi homogeneizada e transferida para vial de injeção para posterior análise em CG-EM.

Para a análise do resíduo de bifentrina na água do tanque de lavagem das bananas, não houve um tratamento estatístico específico. Nas três amostras da água do tanque, foi analisada a presença ou não do resíduo, detectado por cromatografia gasosa, nas condições estabelecidas.

\section{Resultados e discussão}

Na Figura 1, pode-se observar o cromatograma para a solução padrão de bifentrina na concentração de $1 \mathrm{mg} \mathrm{L}^{-1}$, em que o sinal em 9,98 min é atribuído à bifentrina. A solução padrão é utilizada para a obtenção do cromatograma relativo ao analito estudado e das porcentagens de recuperação do analito, nas condições cromatográficas estipuladas.

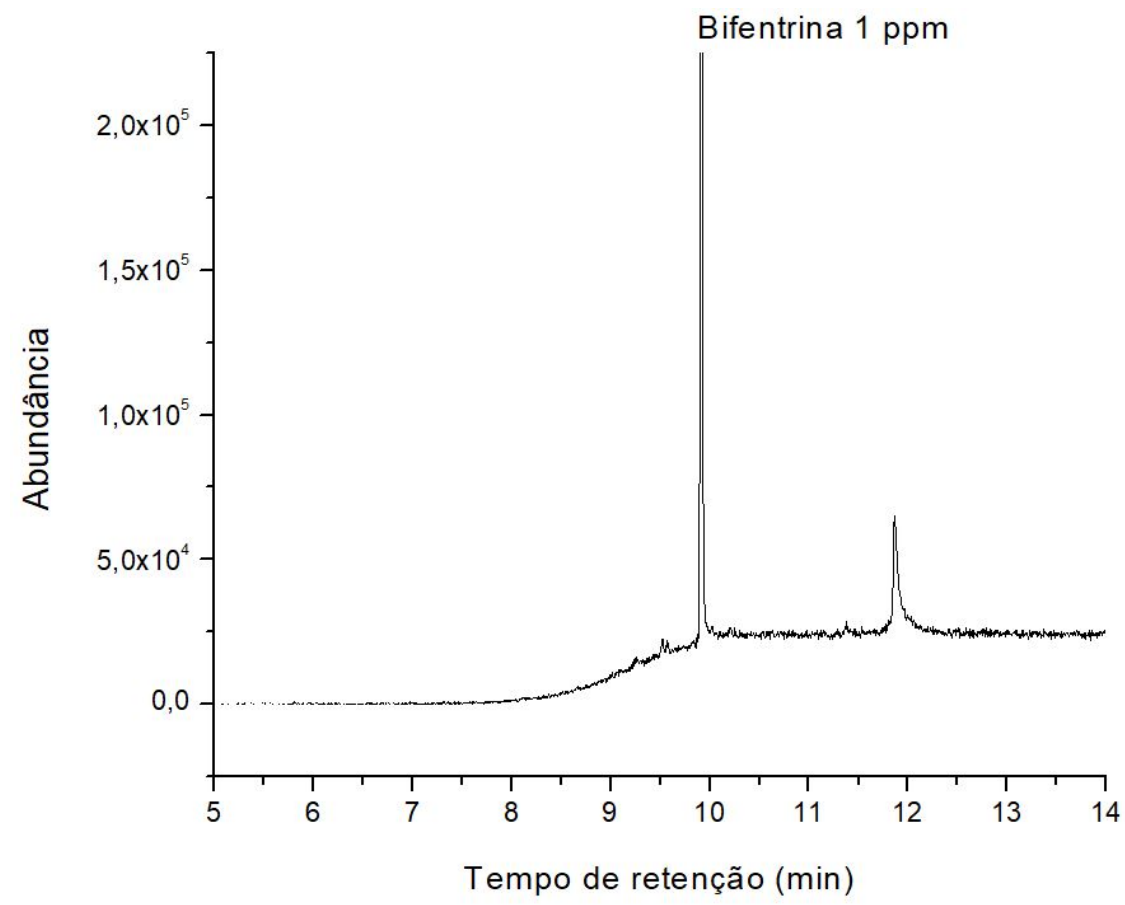

Figura 1. Cromatograma no modo monitoramento de íons seletivo (MIS) da solução padrão de bifentrina a $1 \mathrm{mg} \mathrm{L}^{-1}$. 
As porcentagens de recuperação de bifentrina nas matrizes casca, polpa e casca + polpa de banana estão apresentadas na Tabela 1, na qual se verifica extração de $88,16 \%, 103,4 \%$ e $89,32 \%$, respectivamente. Segundo os critérios de aceitabilidade para resíduo de agrotóxico, estipulados pelo Manual de Garantia da Qualidade Analítica (Brasil, 2011), o procedimento analítico deve ser capaz de recuperar, em cada nível de fortificação e para cada matriz representativa, de $70 \%$ a $120 \%$, em média, para todos os analitos. Neste sentido, verifica-se que a porcentagem de recuperação está dentro dos padrões estabelecidos, ainda que, para polpa da banana, tenha sido superior a 100\%, devido ao efeito matriz.

Tabela 1. Porcentagens de recuperação (\%R) da bifentrina em casca, polpa e casca + polpa de banana 'Grande Naine', pelo método QuEChERS.

\begin{tabular}{ccc}
\hline & Faixa de trabalho $\left(\mu \mathbf{g ~ L}^{-1}\right)$ & \% Recuperação \pm DPR \\
\hline Casca & 50 a 500 & $88,16 \pm 2,5$ \\
\hline Polpa & 50 a 500 & $103,4 \pm 2,7$ \\
\hline Casca + Polpa & 50 a 500 & $89,32 \pm 6,8$ \\
\hline
\end{tabular}

DPR = Desvio Padrão Relativo.

Os componentes da matriz influenciam diretamente na quantificação dos agrotóxicos e é mais significativo em matrizes complexas, como as frutas. Em amostras de goiaba, pigmentos e ácidos orgânicos podem interferir na análise e contribuir para o efeito da matriz (Guedes et al., 2016). Sousa et al. (2012) avaliaram o efeito matriz na análise de 11 agrotóxicos e verificaram que tomate, uva e abacaxi causaram maior efeito matricial quando comparados com amostras de maçã e batata. Silva et al. (2017) concluíram que os agrotóxicos são muito suscetíveis ao efeito matriz promovido por amostras ambientais (água de rio).

Não foi detectado resíduo de bifentrina na polpa dos frutos lavados ou não lavados, no ponto de colheita ou maduros, tampouco nas amostras de água do tanque de lavagem das pencas. Vieira et al. (2007) observaram que a estabilidade dos piretroides em solução aquosa é muito pequena e que, nos primeiros 10 dias do estudo, ocorreu rápida degradação, com perda de aproximadamente $80 \%$ do total. Posteriormente, entre o $11^{\circ}$ e $60^{\circ}$ dia, ocorreu degradação lenta, permanecendo, em média, $3 \%$ de permetrina, cipermetrina, deltametrina e $\lambda$-cialotrina, após 80 dias.

$\mathrm{Na}$ casca e em casca + polpa dos frutos lavados e não lavados, no ponto de colheita, foi verificada a presença de resíduo de bifentrina, sendo estatisticamente diferente em cada matriz, pelo teste $\mathrm{F}$ (Tabela 2).

Tabela 2. Resumo da análise de variância para resíduo de bifentrina em casca e em polpa + casca de banana 'Grande Naine' no ponto de colheita, lavada e não lavada.

\begin{tabular}{cccc}
\hline Fontes de Variação & G.L. & Quadrado médio & Fc \\
\hline Tratamento & 3 & 0,52 & \multirow{2}{*}{$125,92^{*}$} \\
\hline Resíduo & 9 & $4,1 \mathrm{xE}^{-4}$ & \\
\hline $\mathrm{CV}(\%)$ & & 15,49 & \\
\hline
\end{tabular}

$\mathrm{Fc}=$ Fcalculado. *significativo a 5\% pelo teste F. CV $(\%)=$ Coeficiente de Variação. G.L. $=$ Graus de Liberdade.

Os cromatogramas de extratos das amostras de casca e de casca + polpa de banana 'Grande Naine', no ponto de colheita, lavadas e não lavadas, e o do padrão de bifentrina $\left(50 \mu \mathrm{g} \mathrm{L}^{-1}\right)$ estão apresentados na Figura 2. 


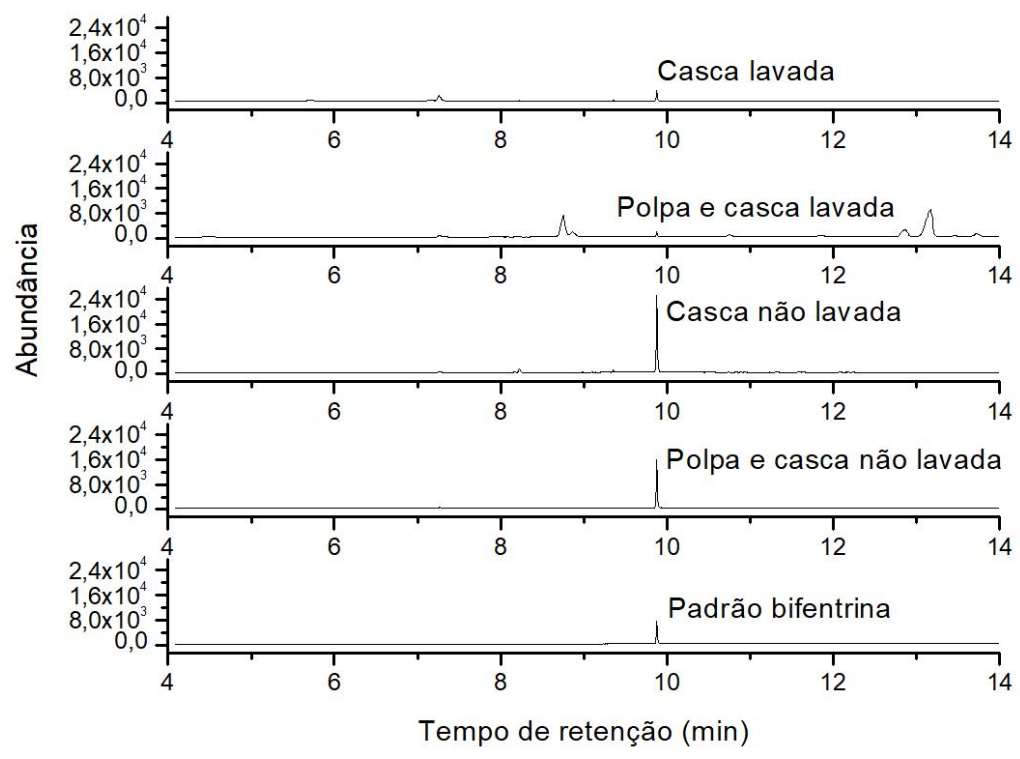

Figura 2. Cromatogramas no modo monitoramento de íons seletivo (MIS) de bifentrina em casca e casca + polpa de banana 'Grande Naine' no ponto de colheita, lavadas e não lavadas, e do padrão bifentrina.

A maior concentração de bifentrina no ponto de colheita foi verificada na casca dos frutos não lavados (Tabela 3), sendo de $0,31 \mathrm{mg} \mathrm{kg}^{-1}$, e valor intermediário ocorreu em casca + polpa $\left(0,16 \mathrm{mg} \mathrm{kg}^{-1}\right)$ dos frutos não lavados. A redução de 51,6\% na concentração do agrotóxico pode ser devida à a diluição promovida pela fração polpa dessa matriz, na qual não foi detectado resíduo. Entre casca e casca + polpa de frutos lavados, não houve diferença significativa, sendo as concentrações de $0,060 \mathrm{mg} \mathrm{kg}^{-1} \mathrm{e} 0,010 \mathrm{mg} \mathrm{kg}^{-1}$, respectivamente. No entanto, na matriz casca + polpa, a concentração de bifentrina foi menor que o LMR preconizado tanto pela ANVISA quanto pelo Codex Alimentarius.

Tabela 3. Resíduo de bifentrina em casca e em casca + polpa de banana 'Grande Naine' no ponto de colheita, lavadas e não lavadas.

\begin{tabular}{ccccc}
\hline & \multicolumn{4}{c}{ Matriz } \\
\cline { 2 - 5 } & Casca lavada & Casca + Polpa lavadas & $\begin{array}{c}\text { Casca não } \\
\text { lavada }\end{array}$ & $\begin{array}{c}\text { Casca + Polpa não } \\
\text { lavadas }\end{array}$ \\
\hline Bifentrina $\left(\mathrm{mg} \mathrm{kg}^{-1}\right)$ & $0,060 \mathrm{C}$ & $0,010 \mathrm{C}$ & $0,31 \mathrm{~A}$ & $0,16 \mathrm{~B}$ \\
\hline
\end{tabular}

Médias seguidas de mesma letra não diferem estatisticamente pelo teste Tukey.

O Limite Máximo de Resíduo (LMR) permitido pela Agência Nacional de Vigilância Sanitária (ANVISA) para aplicação localizada (saco para proteção do cacho) de bifentrina em banana é $0,020 \mathrm{mg} \mathrm{kg}^{-1}$, com intervalo de segurança de um dia (Agência Nacional de Vigilância Sanitária, 2019). Em sua grande maioria, bananas são consumidas maduras e descascadas, mas a biomassa e a farinha são subprodutos elaborados com os frutos ainda verdes e com casca. Portanto, a atenção com a segurança alimentar deve ocorrer independente da forma de consumo.

Cuidados com a presença de compostos acima do LMR são necessários, pois, geralmente, as avaliações de conformidade são realizadas durante a comercialização das frutas, quando ainda não estão aptas ao consumo. Assim, as concentrações devem atender à Legislação do local de destino da fruta, evitando-se as barreiras técnicas comerciais. No comércio internacional, o Codex Alimentarius determina o LMR de agrotóxicos nos produtos de origem animal ou vegetal destinados ao consumo (Hermida et al., 2015) e, para resíduo de bifentrina em banana, o LMR é $0,10 \mathrm{mg} \mathrm{kg}^{-1}$ (Food and Agriculture Organization, 2019). 
O procedimento usual de lavagem das pencas de banana não eliminou a bifentrina por completo na casca dos frutos avaliados no ponto de colheita, mas as concentrações foram inferiores às dos frutos não lavados. Até o amadurecimento, período de 14 dias, o resíduo foi eliminado completamente com a lavagem ou apresentou-se em concentrações abaixo do limite de quantificação e detectável pelo método. Portanto, na casca e em casca + polpa de frutos maduros, somente foi detectado resíduo de bifentrina naqueles não lavados (Figura 3), com diferença significativa entre as concentrações nas diferentes matrizes (Tabela 4). Na casca, foi quantificado $0,10 \mathrm{mg} \mathrm{kg}^{-1}$ de bifentrina, enquanto, em casca + polpa, a concentração foi de $0,040 \mathrm{mg} \mathrm{kg}^{-1}$, valores que correspondem a $32,3 \%$ e $25 \%$ de redução em relação às mesmas matrizes, no ponto de colheita, respectivamente.

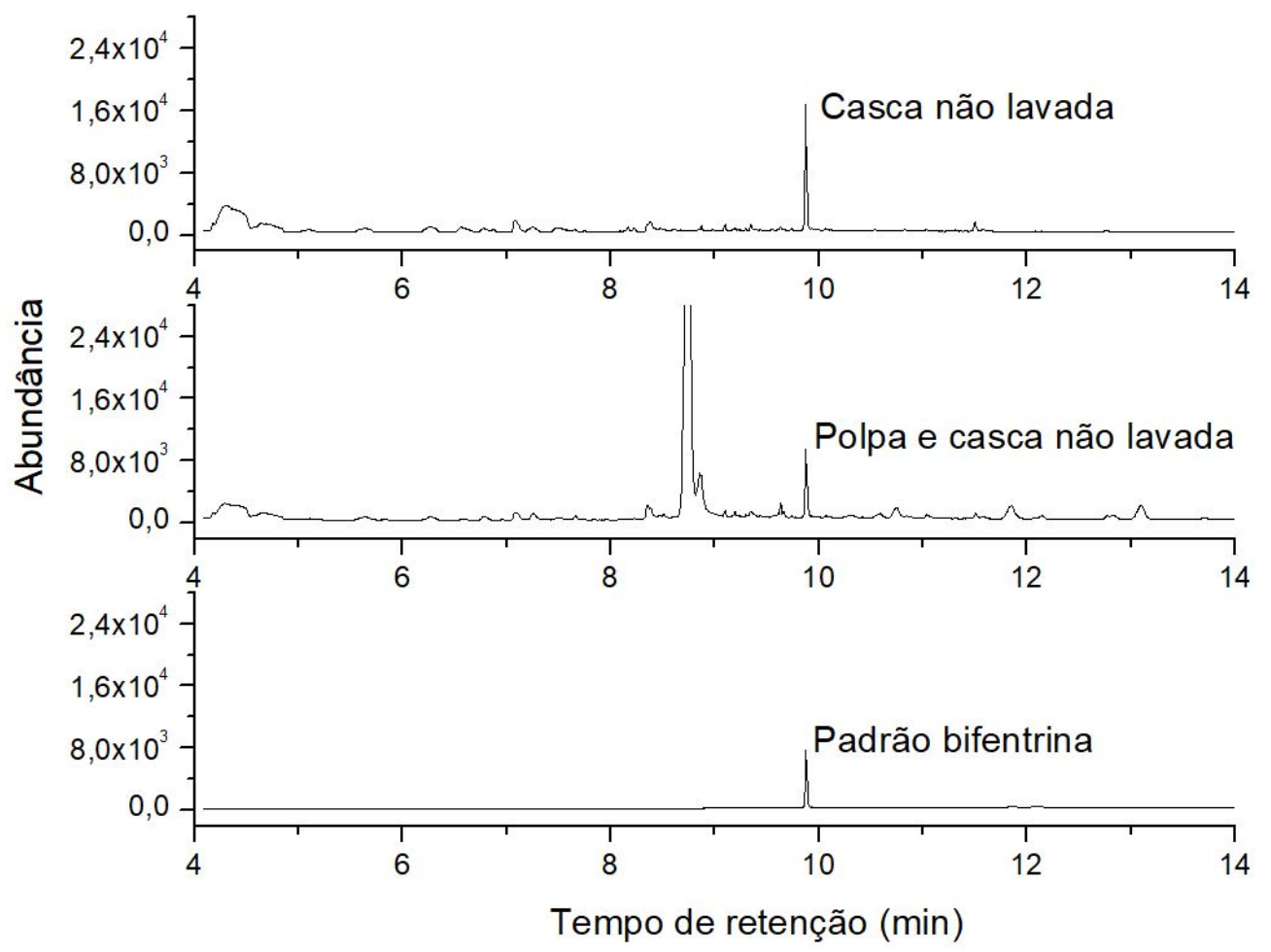

Figura 3. Cromatogramas no modo monitoramento de íons seletivo (MIS) de bifentrina em casca e casca + polpa de banana 'Grande Naine' maduras não lavadas e do padrão bifentrina.

Tabela 4. Resumo da análise de variância para resíduo de bifentrina em casca e em polpa + casca de banana 'Grande Naine' madura, não lavada.

\begin{tabular}{cccc}
\hline Fontes de Variação & G.L. & Quadrado médio & Fc \\
\hline Tratamento & 1 & $4,8 \times \mathrm{E} \mathrm{E}^{-3}$ & $55,58^{*}$ \\
\hline Resíduo & 5 & $8,7 \mathrm{E}^{-5}$ & \\
\hline $\mathrm{CV}(\%)$ & & 12,99 & \\
\hline
\end{tabular}

$\mathrm{Fc}=$ Fcalculado. $*$ significativo a $5 \%$ pelo teste $\mathrm{F}$.

Além da 'limpeza' que a lavagem promoveu, contribuindo para redução do resíduo, a degradação do composto durante o amadurecimento tem sido a provável causa da redução das concentrações. A luz, o pH, a temperatura e o tempo de armazenamento são variáveis que interferem na degradação dos piretroides, conforme verificado por Silveira et al. (2006) e Santos et al. (2007). 


\section{Conclusões}

Em frutos no ponto de colheita, lavados e não lavados, foi quantificado resíduo de bifentrina na casca e em casca + polpa, sendo as maiores concentrações na casca das bananas não lavadas.

Em frutos maduros, a bifentrina foi quantificada na casca e em casca + polpa, mas somente daqueles não lavados.

Não foi detectado resíduo de bifentrina na polpa dos frutos, seja no ponto de colheita ou maduros, lavados ou não lavados, nem na água do tanque de lavagem.

\section{Agradecimentos}

À (FAPEMIG), pela concessão de bolsa de estágio pós-doutoral à primeira autora, ao Conselho Nacional de Desenvolvimento Científico e Tecnológico (CNPq) (processos 420637/2016-8 e 308480/2019-8), à Coordenação de Aperfeiçoamento de Pessoal de Nível Superior (CAPES), ao Instituto de Ciências AgráriasUFMG (ICA-UFMG) e à Universidade Federal de Minas Gerais (UFMG), pela infraestrutura e execução deste trabalho.

\section{Referências}

Agência Nacional de Vigilância Sanitária - ANVISA. (2019). Monografias autorizadas: Índice Monográfico Nome B26 Bifentrina. Recuperado em 12 de novembro de 2019, de http://portal.anvisa.gov.br/registros-eautorizacoes/agrotoxicos/produtos/monografia-de-agrotoxicos/autorizadas

Brasil. Ministério da Agricultura Pecuária e Abastecimento Secretaria de Defesa Agropecuária. (2011). Manual de garantia da qualidade analítica. Brasília: MAPA/ACS.

Brasil. Ministério da Agricultura Pecuária e Abastecimento. Coordenação-Geral de Agrotóxicos e Afins/DFIA/SDA. (2019). Agrofit: Sistema de agrotóxicos fitossanitários. Brasília. Recuperado em 29 de outubro de 2019, de http://agrofit.agricultura.gov.br/agrofit_cons/principal_agrofit_cons

Carneiro, R., Oliveira, F. A. S., Madureira, F. D., Silva, G., Souza, W. R., \& Lopes, R. P. (2013). Development and method validation for determination of 128 pesticides in bananas by modified QuEChERS and UHPLC MS/MS analysis. Food Control, 33(2), 413-423. http://dx.doi.org/10.1016/j.foodcont.2013.02.027

Castricini, A., Oliveira, A. L. S., Pinho, G. P., Rodrigues, M. G. V., \& Silvério, F. O. (2019). Pyrethroid insecticide residue in 'Grande Naine' banana peel and pulp during maturation. Food Science and Technology, 39(Supl.1), 68-73. http://dx.doi.org/10.1590/fst.37117

Companhia de Entrepostos e Armazéns Gerais de São Paulo - CEAGESP. Programa Brasileiro para a Modernização da Horticultura \& Produção Integrada de Frutas - PBMH \& PIF. (2006). Normas de classificação de banana (Documentos, No. 29). São Paulo: CEAGESP.

Freitas, R. S. (2013). Ozônio na degradação de resíduos de inseticidas em grãos de milho (Tese de doutorado). Universidade Federal de Viçosa, Viçosa.

Guedes, J. A. C., Silva, R. O., Lima, C. G., Milhome, M. A. L., \& Nascimento, R. F. (2016). Matrix effect in guava multiresidue analysis by QuEChERS method and gas chromatography coupled to quadrupole mass spectrometry. Food Chemistry, 199, 380-386. PMid:26775985. http://dx.doi.org/10.1016/j.foodchem.2015.12.007

Hermida, C., Pelaez, V., \& Silva, L. (2015). Limites de resíduos de agrotóxicos e barreiras técnicas comerciais. Agroalimentaria, 21(41), 151-170. Recuperado em 1 de setembro de 2020, de https://dialnet.unirioja.es/servlet/articulo?codigo=6318168

<eref>Instituto Brasileiro de Geografia e Estatística - IBGE. SIDRA. (2018). Produção agrícola municipal. Recuperado em 25 de outubro de 2019, de https://sidra.ibge.gov.br/Tabela/1613</eref>

Miyamoto, J., Kaneko, H., Tsuji, R., \& Okuno, Y. (1995). Pyrethroids, nerve poisons: How their risks to human health should be assessed. Toxicology Letters, 82-83(1), 933-940. PMid:8597164. http://dx.doi.org/10.1016/0378-4274(95)03604-0

Oviedo, M. T. P., Toledo, M. C. de F., \& Vicente, E. (2003). Resíduos de agrotóxicos piretróides em hortaliças. Pesticidas: Revista de Ecotoxicologia e Meio Ambiente, 13(1), 9-18. http://dx.doi.org/10.5380/pes.v13i0.3160

Universidade Federal de Viçosa - UFV. Sistema para Análises Estatísticas - SAEG. (2007). SAEG 9.1. Viçosa: Fundação Arthur Bernardes.

Silva, V. P. A., Paz, M. S. O., Cavalcante, R. M., \& Nascimento, R. F. (2017). Strategy for correction of matrix effect on the determination of pesticides in water bodies using SPME-GC-FID. Journal of the Brazilian Chemical Society, 28(6), 1081-1090. http://dx.doi.org/10.21577/0103-5053.20160264

Silveira, R. D., Faroni, L. R. A., Pimentel, M. A. G., Peternelli, L. A., \& Zocolo, G. J. (2006). Eficácia biológica e persistência de bifentrina pulverizada em grãos de milho com diferentes temperaturas. Neotropical Entomology, 35(2), 264-268.

PMid:17348140. http://dx.doi.org/10.1590/S1519-566X2006000200017 
Silvério, F. O., Silva, J. G. S., Aguiar, M. C. S., Cacique, A. P., \& Pinnho, G. P. (2012). Análise de agrotóxicos em água usando extração líquido-líquido com partição em baixa temperatura por cromatografia líquida de alta eficiência. Química Nova, 35(10), 2052-2056. http://dx.doi.org/10.1590/S0100-40422012001000027

Sousa, F. A., Costa, A. O. G., Queiroz, M. E. L. R., Teófilo, R. F., Neves, A. A., \& Pinho, G. P. (2012). Evaluation of matrix effect on the GC response of eleven pesticides by PCA. Food Chemistry, 135(1), 179-185. http://dx.doi.org/10.1016/j.foodchem.2012.04.063

Tiwari, B. K., Brennan, C. S., Curran, T., Gallagher, E., Cullen, P. J., \& O’Donnell, C. P. (2010). Application of ozone in grain processing. Journal of Cereal Science, 51(3), 248-255. http://dx.doi.org/10.1016/j.jcs.2010.01.007

Vieira, H. P., Neves, A. A., \& Queiroz, M. E. L. (2007). R de. Otimização e validação da técnica de extração líquido-líquido com partição em baixa temperatura (ell-pbt) para piretróides em água e análise por CG. Química Nova, 30(3), 535-540. http://dx.doi.org/10.1590/S0100-40422007000300006

Santos, M. A. T., Areas, M. A., \& Reyes, F. G. R. (2007). Piretróides: Uma visão geral. Alimentos e Nutrição, 18(3), 339-349. Recuperado em 1 de setembro de 2020, de https://www.researchgate.net/publication/49599762_Piretroides__uma_visao_geral

Food and Agriculture Organization - FAO. CODEX ALIMENTARIUS. International Foods Standards. (2019). Pesticide Index: 178 - Bifenthrin. Recuperado em 12 de novembro de 2019, de http://www.fao.org/fao-who-codexalimentarius/codextexts/dbs/pestres/pesticide-detail/en/?p_id=178

Wakil, W., Riasat, T., \& Lord, J. C. (2013). Effects of combined thiamethoxam and diatomaceous Earth on mortality and progeny production of four Pakistani populations of Rhyzopertha dominica (Coleoptera: Bostrichidae) on wheat, rice and maize. Journal of Stored Products Research, 52(1), 28-35. http://dx.doi.org/10.1016/j.jspr.2012.09.002

Financiamento: Ministério da Ciência, Tecnologia e Inovação/Conselho Nacional de Desenvolvimento Científico e Tecnológico (processos 420637/2016-8 e 308480/2019-8); Coordenação de Aperfeiçoamento de Pessoal de Nível Superior; Fundação de Amparo à Pesquisa do Estado de Minas Gerais 